\title{
A Stochastic Programming Approach For Multi-Period Portfolio Optimization With Transaction Costs
}

\author{
Mehmet Can ${ }^{\mathrm{a}}$, Narela Bajram ${ }^{\mathrm{b}}$ \\ ${ }^{\mathrm{a}}$ Faculty of Engineering, International University of Sarajevo, \\ Hrasnička Cesta 15, 71200 Sarajevo \\ Bosnia and Herzegovina, \\ mcan@ius.edu.ba \\ ${ }^{\mathrm{b}}$ Faculty of Management, International University of Sarajevo, \\ Hrasnička Cesta 15, 71200 Sarajevo \\ Bosnia and Herzegovina, \\ narela@ius.edu.ba
}

\begin{abstract}
This paper uses stochastic programming to solve multi-period investment problems. We combine the feature of asset return predictability with practically relevant constraints arising in a multi-period investment context. The objective is to maximize the expected utility of the returns the periods to balance the liabilities. Asset returns and state variables follow a firstorder vector auto-regression and the associated uncertainty is described by discrete scenario trees. To deal with the long time intervals involved in multi-period problems, we consider short-term decisions, and incorporate a solution for the long, subsequent steady-state period to account for end effects.
\end{abstract}

Keywords - Portfolio optimization, multi- period asset allocation, stochastic programming, Scenario trees, transaction costs 


\section{INTRODUCTION}

The classical treatments of strategic asset allocation can be traced back to Samuelson (1969) and Merton (1969, 1971). In the light of Markowitz' seminal papers on single-period portfolio selection, the early literature focused on conditions leading to the optimality of myopic policies, i.e., conditions under which portfolio decisions for multi-period problems coincide with those for single period problems. In addition, the lack of computing power lead to formulate models driven by the quest for closed form solutions. To achieve these objectives, rather restrictive assumptions were made, and many of these models' results turned out to be inconsistent with conventional wisdom as expressed by the so-called Samuelson puzzle: whereas one of the main results from early multi-period portfolio models is that the fractions of risky assets are constant over time, this contradicts the advice obtained from many professionals in practice that investors should hold a share of risky assets which declines steadily as they approach retirement (often called the age effect). Since then, many researchers have tried to resolve this puzzle which is mainly rooted in some of the (simplifying) assumptions used in early models (fixed planning horizon, time-constant investment opportunities, no intermediate consumption, etc.).

Research in the area of life-cycle asset allocation models regained momentum in the early 1990s for two main reasons: first, a number of economic factors increased the number of people with sizeable wealth to invest (the "generation of heirs"), coupled with increased uncertainty about the security of public pension systems. Second, the enormous increase in computer power enabled the solution of models with more realistic assumptions. A number of additional features have been added to the classical models, in many cases with the goal of resolving the Samuelson puzzle: stochastic labor income, time-varying investment opportunities, parameter uncertainty (with and without learning), special treatment of certain asset classes (real estate), and habit formation, to name just the most important developments.

In contrast to other approaches in the literature using nonlinear optimization (see, e.g., Blomvall and Lindberg 2002; Gondzio and Grothey 2007), we use multi-period stochastic linear programming (SLP) to solve the problem of optimal life-cycle asset allocation and consumption. This method has been explicitly chosen with the practical application of the approach in mind. Many features which are considered important for investment decisions in practice can be easily incorporated when using SLP. For example, personal characteristics of the investor can be taken into account (e.g., mortality risk, risk attitude, retirement, future cash flows for major purchases or associated with other life events). Combined with the availability of efficient solvers, this explains why the SLP approach has been successfully applied to a wide range of problems (see, e.g., Wallace and Ziemba 2005). To nest classical analytical results from this area within our model, we maximize expected utility of consumption over the investor's lifetime and expected utility of bequest rather than other objectives which can be implemented more easily (e.g., piecewise linear or quadratic penalty functions, or minimizing $\mathrm{CVaR}$ ).

The paper is organized as follows: in Sect. 2 we provide a classification of the more recent life-cycle asset allocation models based on the type of available solutions. Section 3 describes the stochastic programming model, in particular the formulation of the objective, the optimization approach for its linearization, and the generation of scenarios. In Sect. 4 results from the SLP are compared to those in Campbell et al. (2003), and results for an extended setting are presented. Section 5 concludes.

\section{OVERVIEW OF SOLUTION METHODS}

Many papers try to extend the classic Merton framework along different lines while maintaining analytical solutions (see, e.g., Bodie et al. 1992; Balvers and Mitchell 1997; Kim and Omberg 1996; Wachter 2002; Liu 2007). Analytical solutions are available for restrictive assumptions on the utility structure and the planning horizon.

Another set of models obtain solutions which are exact only under (generally less stringent) assumptions, and approximately correct if these assumptions are not exactly met. In some cases, these approximate solutions are available in closed form, while others must be solved numerically. Approximate analytical solutions are provided by, e.g., Campbell and Viceira (1999, 2001, 2002), Campbell et al. (2004), and Chacko and Viceira (2005). Approximate numerical solutions can be found in, e.g., Schroder and Skiadas (1999) and Campbell et al. (2003).

To give some examples for the restrictive assumptions mentioned above, a number of the models from this category assume either a deterministic or an infinite planning horizon. Some of the finite-horizon models define utility over terminal wealth only. These assumptions are clearly problematic for individuals who face an uncertain lifetime and derive their utility mainly from what they consume during their lives, and not only from their bequest.

An important reference for the present paper is Campbell et al. (2003). They model asset returns and state variables as a first-order vector autoregression $\operatorname{VAR}(1)$ and consider Epstein-Zin utility with an infinite planning horizon. Additional assumptions include the absence of borrowing and short-sale constraints. Linearizing the portfolio return, the budget constraint, and the Euler equation, they arrive at a system of linear-quadratic equations for portfolio weights and consumption as functions of state variables. This system of equations can be solved analytically, yielding solutions which are exact only for a special case (very short time intervals and elasticity of intertemporal substitution equal to one), and accurate approximations in its neighborhood.

In Sect. 4, we replicate their results as far as possible and subsequently exemplify the application of the SLP approach by investigating aspects beyond the scope of their setting, such as constraints on asset weights, transaction costs, and labor income. 
Two main types of numerical solution methods can be found in the literature: One approach works via grid methods discretizing the state space, the other is based on Monte Carlo simulation. Grid discretizations are used in, among others, Brennan et al. (1997), Barberis (2000), Campbell et al. (2001), Cocco et al. (2005), and Gomes and Michaelides (2005). The main drawback of this approach is that the reduction in the state-space dimensionality, which is crucial for the solution in terms of computation time, requires to restrict the investment opportunity set (usually to one risky and one riskless asset). This may be inappropriate for many investors. Detemple et al. (2003) and Brandt et al. (2005) use simulation-based approaches.Detemple et al. approximate deviations from a closed-form solution, while Brandt et al. provide an approach that is inspired by the option pricing algorithm by Longstaff and Schwartz (2001).

The SLP used in the present paper has been applied successfully to a number of related problems. To cite only a few examples, there are applications in insurance (Cariño and Ziemba 1994, 1998; Cariño et al. 1998), and the pension fund industry (e.g., Gondzio and Kouwenberg 2001). Zenios (1999) surveys large-scale applications of SLP to fixed income portfolio management. General aspects of applying such models in a strategic asset allocation context are discussed in Ziemba and Mulvey (1998)), Pflug and Swietanowski (2000), Gondzio and Kouwenberg (2001), Wallace and Ziemba (2005), and Geyer and Ziemba (2007). Particular aspects that are relevant in a life-cycle portfolio context are discussed in Geyer et al. (2007).

\section{A MULTISTAGE MODEL: ASSET- LIABILITY MANAGEMENT}

The best way to introduce multistage stochastic models is a simple asset liability management (ALM) model (Birge, and Louveaux 1967). We have an initial wealth $W^{0}$, that should be properly invested in such a way to meet a liability $\mathrm{L}$ at the end of the planning horizon $\mathrm{H}$. If possible, we would like to own a terminal wealth $W^{H}$ larger than L; however, we should account properly for risk aversion, since there could be some chance to end up with a terminal wealth that is not sufficient to pay for the liability, in which case we will have to borrow some money. A nonlinear, strictly concave utility function of the difference between the terminal wealth $W^{H}$, which is a random variable, and the liability $L$ would do the job, but this would lead to a nonlinear programming model.

As an alternative, we may build a piecewise linear utility function like the one illustrated in Fig. 1.

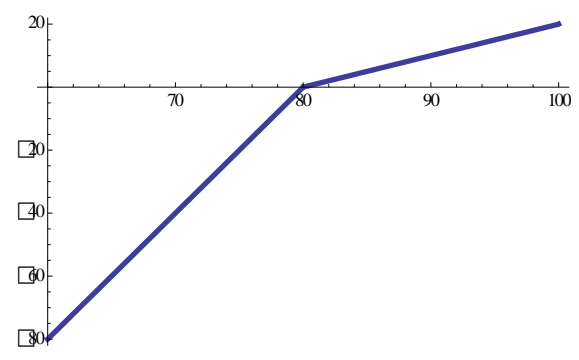

Fig. 1 Piecewise linear utility function
The utility is zero when the terminal wealth $W^{H}$ matches the liability exactly. If the slope $\mathrm{r}$ penalizing the shortfall is larger than q, this function is concave (but not strictly).

The portfolio consists of a set of I assets. For simplicity, we assume that we may rebalance it only at a discrete set of time instants $\mathrm{t}=1, \ldots, \mathrm{H}-1$, with no transaction cost; the initial portfolio is chosen at time $t=0$, and the liability must be paid at time $\mathrm{H}$. Time period $\mathrm{t}$ is the period between time instants $\mathrm{t}$ 1 and $t$. In order to represent uncertainty, we may build a tree like that in Fig. 1 (Høyland K, Wallace SW 2001, Pflug GC 2001). Each node $n_{k}$ in the tree corresponds to an event, where we should make some decision. We have an initial node $n_{0}$ corresponding to time $\mathrm{t}=0$.

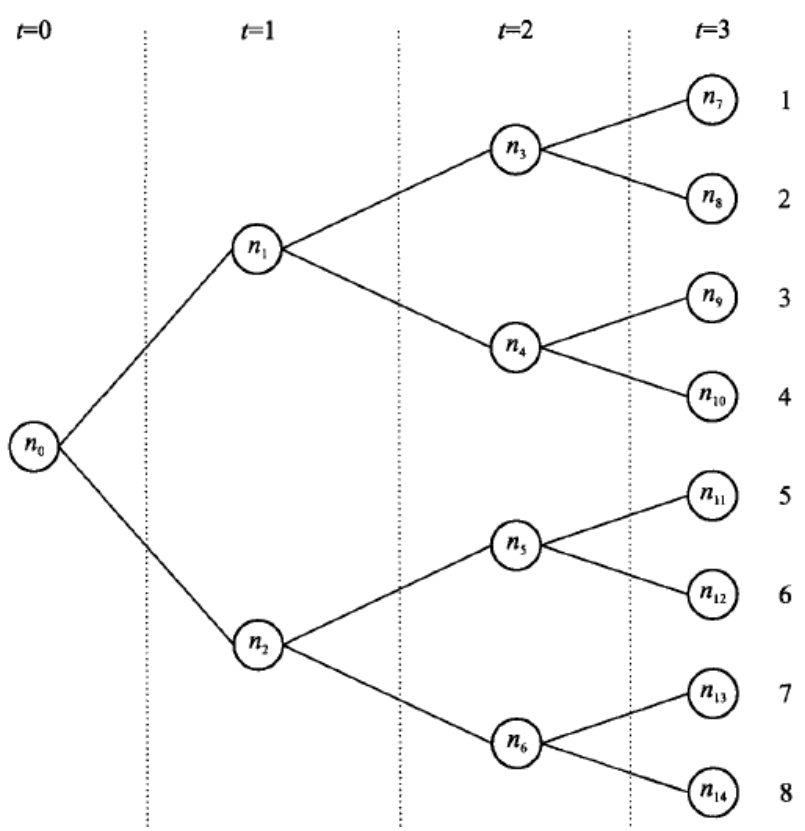

Fig. 2 Scenario tree for a simple asset- liability management problem.

Then, for each event node, we have two branches; each branch is labeled by a conditional probability of occurrence, $P\left(n_{k} \mid n_{i}\right)$, where $n_{i}=a\left(n_{k}\right)$ is the immediate predecessor of node $n_{k}$. Here, we have two nodes at time $\mathrm{t}=1$ and four at time $t=2$, where we may rebalance our portfolio on the basis of the previous asset returns. Finally, in the eight nodes corresponding to $t=3$, the leaves of the tree, we just compare the terminal wealth with the liability and evaluate the utility function. Each node of the tree is associated with the set of asset returns during the corresponding time period. A scenario consists of an event sequence, i.e., a sequence of nodes in the tree, along with the associated asset returns. We have 8 scenarios in Fig. 2. For instance, scenario 2 consists of the node sequence $n_{3}, n_{4}, n_{5}, n_{6}$. The probability of each scenario depends on the conditional probability of each node on its path (Samuelson PA 1969).

If each branch at each node is equiprobable, i.e., the conditional probabilities are always, each scenario in the figure has probability $\pi^{s}=1 / 8$, for $s=1, \ldots, 8$. The branching factor may be arbitrary in principle; the more branches we use, 
the better our ability to model uncertainty; unfortunately, the number of nodes grows exponentially with the number of stages, as well as the computational effort.

At each node in the tree, we must make a set of decisions. In practice, we are interested in the decisions that must be implemented here and now, i.e., those corresponding to the first node of the tree; the other (recourse) decision variables are instrumental to the aim of devising a robust plan, but they are not implemented in practice, as the multistage model is solved on a rolling-horizon basis. This suggests that, in order to model the uncertainty as accurately as possible with a. limited computational effort, a possible idea is to branch many paths from the initial node, and less from the subsequent nodes. Each decision at each stage may depend on the information gathered so far, but not on the future; this requirement is called a. nonanticipativity condition. Essentially, this means that decisions made at time t must be the same for scenarios that cannot be distinguished at time $t$. To build a model ensuring that the decision process makes sense, there are two choices:

- We can introduce a set of decision variables $x_{i t}^{s}$, representing wealth allocated to asset $\mathrm{i}$ at time t on scenario s; we should force decision variables to take the same value when appropriate, by writing explicit nonanticipativity constraints for scenarios that cannot be distinguished at time $t$.

- We can associate decision variables with nodes in the scenario trees and write the model in a. way that relates each node to its predecessors.

\section{A TWO-STAGE, THREE ASSETS MODEL: ASSET- LIABILITY MANAGEMENT}

We will illustrate the second alternative in detail, using the following numerical data:

- $\quad$ The initial wealth is $W^{0}=50$.

- $\quad$ The target liability is $L^{S}=100$.

- There are three assets, say, stocks A and B, and bonds; hence, $I=3$.

- In the scenario tree of Fig. 2 we have up - and downbranches; in the (lucky) upbranches, total return is 1.28 for stocks A, 1.40 for stocks B and 1.20 for bonds; in the (bad) downbranches, total return is 1.08 for stocks A, 0.99 for stocks B and 1.12 for bonds (Barberis NC 2000). We see that bonds play the role of safer assets, and stocks B are very risky assets here. We also see that returns are a sequence of i.i.d. random variables, but more realistic scenarios can be defined.

- $\quad$ The reward rate $q$ for excess wealth above the target liability is 1 .

- The penalty rate $r$ for the shortfall below the target liability is 4 .

Let us introduce the following notation:

- $\quad \mathcal{N}$ is the set of event nodes; in our case

$$
\mathcal{N}=\left\{n_{0}, n_{1}, n_{2}, \ldots, n_{14}\right\}
$$

- Each node $n \in N, n \neq n_{0}$, apart from the root node $n_{0}$, has a unique direct predecessor node, denoted by $a(n)$ : for instance, $a\left(n_{3}\right)=n_{1}$.
- $\quad$ There is a set $\mathcal{S} \subset \mathcal{N}$ of leaf (terminal) nodes; in our case

$\mathcal{S}=\left\{n_{7}, n_{8}, n_{9}, \ldots, n_{14}\right\}$,

- For each node $s \in \mathcal{S}$ we have surplus and shortfall variables $w_{+}^{s}$ and $w_{-}^{s}$, related to the difference between terminal wealth and liability.

- $\quad$ There is a set $\mathcal{T} \subset \mathcal{N}$ of intermediate nodes, where portfolio rebalancing may occur after the initial allocation in node no; in our case $\mathcal{T}=\left\{n_{1}, n_{2}, \ldots, n_{6}\right\}$

- For each node $n \in \mathcal{T} \cup n_{0}$ there is a decision variable $x_{i}^{n}$, expressing the money invested in asset $\mathrm{i}$ at node $\mathrm{n}$.

With this notation, the model may be written as follows:

$\max \sum_{s \in \mathcal{S}} \pi^{s}\left(q w_{+}^{S}-\mathrm{r} w_{-}^{s}\right)$

Such that

$\sum_{i=1}^{I} x_{i}^{n_{0}}=W^{0}$

$\sum_{i=1}^{I} R_{i}^{n} x_{i}^{a(n)}=\sum_{i=1}^{I} x_{i}^{n}, \quad \forall n \in \mathcal{T}$

$\sum_{i=1}^{I} R_{i}^{S} x_{i}^{a(s)}=L^{s}+w_{+}^{s}-w_{-}^{s}, \forall s \in \mathcal{S}$

$x_{i}^{n}, w_{+}^{s}, w_{-}^{s} \geq 0$

where $R_{i}^{n}$ is the total return for asset i during the period that leads to node $\mathrm{n}$, and $\pi^{s}$ is the probability of reaching the terminal node $s \in \mathcal{S}$; this probability is the product of all the conditional probabilities on the path that leads from root node no to leaf node $\mathrm{s}$.

Let us choose a nonlinear utility function, such that the objective of the optimization problem becomes:

$\sum_{s \in \mathcal{S}} \pi^{s}\left(\left(w_{+}^{s}\right)^{2}-\left(w_{-}^{s}\right)^{2}\right)$

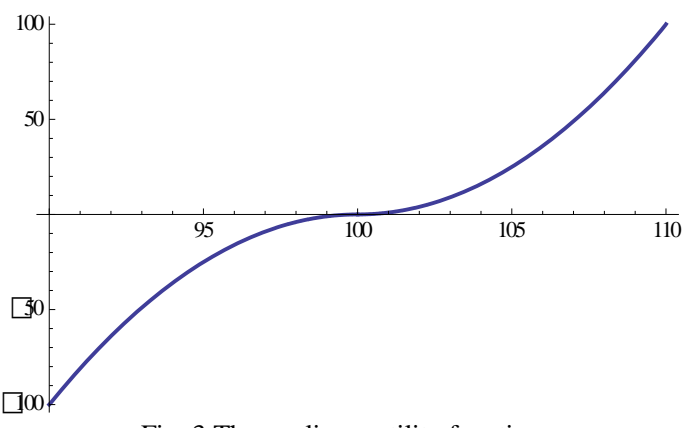

Fig. 3 The nonlinear utility function

In this case we get a better diversification:

Table 1. Investment strategy for a simple ALM problem with nonlinear utility functions.

\begin{tabular}{|l|r|r|r|}
\hline Node & \multicolumn{1}{|l|}{ Stocks A } & \multicolumn{1}{l|}{ Stocks B } & \multicolumn{1}{l|}{ Bonds } \\
\hline n0 & 0 & 50 & 0 \\
\hline n1 & 0 & 70 & 0 \\
\hline n2 & 14.34 & 12.44 & 22.71 \\
\hline n3 & 0 & 98 & 0 \\
\hline n4 & 37.34 & 0 & 31.96 \\
\hline n5 & 56.60 & 0 & 6.43 \\
\hline n6 & 1.86 & 23.21 & 28.18 \\
\hline
\end{tabular}




\section{ASSET-LIABILITY MANAGEMENT WITH TRANSACTION COSTS}

To give the reader an idea of how to build nontrivial financial planning models, we generalize a bit the model formulation of the previous section, in order to account for proportional transaction costs. The assumptions and the limitations behind this extended model are the following:

- We are given a set of initial holdings for each asset; this is a more realistic assumption, since we should use the model to rebalance the portfolio periodically, according to a rolling-horizon strategy.

- We take proportional (linear) transaction costs into account; the transaction cost is a percentage c of the traded value, for both buying and selling an asset.

- We want to maximize the expected utility of the terminal wealth.

- There is a stream of uncertain liabilities that we have to meet.

- We do not consider the possibility of borrowing money; we assume that all of the available wealth at each rebalancing period is invested in the available assets; actually, the possibility of investing in a riskfree asset is implicit in the model.

- We do not consider the possibility of investing new cash at each rebalancing date (as would be the case, e.g. for a pension fund).

Some of the limitations of the model may easily be relaxed. The important point we make is that when transaction costs are involved, we have to introduce new decision variables to express the amount of assets (number of shares, not the monetary value) held, sold, and bought at each rebalancing date. We use a notation which is similar to that used in the previous ALM formulation:

- $\mathcal{N}$ is the set of nodes in the tree; $n_{0}$ is the root node.

- $\quad$ The (unique) predecessor of node $n \in \mathcal{N}, n \neq n_{0}$, is denoted by $a(n)$; the set of terminal nodes is denoted by $\mathcal{S}$; as in the previous formulation, each of these nodes corresponds to a scenario, which is the sequence of event nodes along the unique path leading from $n_{0}$ to $s \in \mathcal{S}$, with probability $\pi^{s}$.

- $\mathcal{T}=\mathcal{N} \backslash\left(\left\{n_{0}\right\} \cup \mathcal{S}\right)$ is the set of intermediate trading nodes.

- $L^{n}$ is the liability we have to meet in node $n \in N$; liabilities are node dependent and stochastic.

- $\quad c$ is the percentage transaction cost.

- $\bar{h}_{i}^{n_{0}}$ is the initial holding for asset $i=1, \ldots, I$ at the root node.

- $\quad P_{i}^{n}$ is the price for asset $i$ at node $n$.

- $z_{i}^{n}$ is the amount of asset i purchased at node $n$.

- $y_{i}^{n}$ is the amount of asset $\mathrm{i}$ sold at node $\mathrm{n}$.

- $x_{i}^{n}$ is the amount of asset $\mathrm{i}$ we hold at node $\mathrm{n}$, after rebalancing.

- $W^{s}$ is the wealth at terminal node $s \in \mathcal{S}$.
- $\quad u(W)$ is the utility for wealth $W$; this function is used to express utility of terminal wealth.

On the basis this notation, we may write the following model:

$\max \sum_{s \in \mathcal{S}} \pi^{s} u\left(W^{s}\right)$

Such that

$x_{i}^{n_{0}}=\bar{h}_{i}^{n_{0}}+z_{i}^{n_{0}}-y_{i}^{n_{0}}, i=1, \ldots, I$

$x_{i}^{n}=x_{i}^{a(n)}+z_{i}^{n}-y_{i}^{n}$

$(1-c) \sum_{i=1}^{I} P_{i}^{n} y_{i}^{n}-(1+c) \sum_{i=1}^{I} P_{i}^{n} z_{i}^{n}=L^{n}$,

$\forall n \in \mathcal{T} \cup n_{0}$

$W^{s}=\sum_{i=1}^{I} P_{i}^{s} x_{i}^{a(s)}-L^{s}, \forall s \in \mathcal{S}$

$x_{i}^{n}, y_{i}^{n}, z_{i}^{n}, W^{s} \geq 0$

The objective (8) is the expected utility of the terminal wealth; if we approximate this nonlinear concave function by a piecewise linear concave function, we get an LP problem. Equation (9) expresses the initial asset balance, taking the current holdings into account; the asset balance at intermediate trading dates is taken into account by Eq. (10). Eq. (11) ensures that enough cash is generated by selling assets in order to meet the liabilities; we may also reinvest the proceeds of what we sell in new asset holdings; note how the transaction costs are expressed for selling and purchasing. Eq. (12) is used to evaluate terminal wealth at leaf nodes; note here that we have not taken into account the need to sell assets in order to generate the cash required by the last liability; but this would make only sense if the whole fund is liquidated at the end of the planning horizon. If so, we could rewrite Eq. (12) as

$W^{s}=(1-c) \sum_{i=1}^{I} P_{i}^{s} x_{i}^{a(s)}-L^{s}, \forall s \in \mathcal{S}$

In practice, we would repeatedly solve the model on a rollinghorizon basis, so the exact expression of the objective function is a bit debatable. The role of terminal utility is just to ensure that we are left in a good position at the end of the planning horizon. Let us choose a nonlinear utility function. Then the objective of the optimization problem is:

$\sum_{s \in \mathcal{S}} \pi^{s}\left(W^{s}\right)^{2 / 3}$

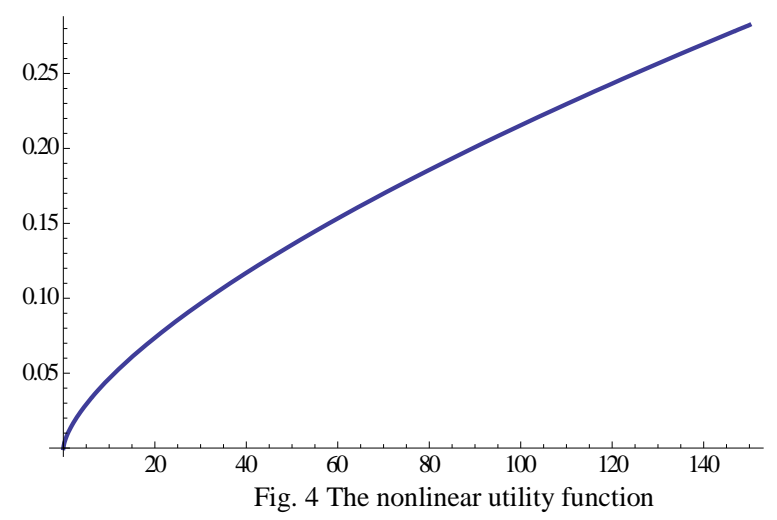

In this case we get a better diversification: 
Table 2. Investment strategy for a simple ALM problem with transaction costs, and with nonlinear utility functions.

\begin{tabular}{|l|l|l|}
\hline Node & Stocks & Bonds \\
\hline n0 & 738.591 & 0.006 \\
\hline n1 & 0.006 & 375.375 \\
\hline n2 & 692.685 & 0.002 \\
\hline n3 & 0.010 & 371.949 \\
\hline n4 & 0.032 & 351.634 \\
\hline n5 & 0.023 & 324.821 \\
\hline n6 & 525.391 & 47.920 \\
\hline
\end{tabular}

\section{CONCLUSION}

The most important point is that we have assumed that the liabilities must be met. This may be a very hard constraint; if extreme scenarios are included in the formulation, it may well be the case that the model above is infeasible. Therefore, the formulation should be relaxed in a sensible way; we could consider the possibility of borrowing cash; we could also introduce suitable penalties for not meeting the liabilities.

In principle, we could also require that the probability of not meeting the liabilities is small enough; this leads to chanceconstrained formulations, for which we refer the reader to the literature (Campbell JY, Viceira LM 2002, Heitsch H, Römisch W 2003, Hochreiter R, Pflug GC 2007, Klaassen P 2002, Liu J 2007, Wallace SW, Ziemba WT (eds) 2005).

\section{REFERENCES}

Barberis NC (2000) Investing for the long run when returns are predictable. J Finance 55: pp 225-264

Birge J R, Louveaux F (1967) Introduction to stochastic programming, Springer Verlag, New York: pp 20-28.

Campbell JY, Viceira LM (2002) Strategic asset allocation. Oxford University Press, USA

Heitsch H, Römisch W (2003) Scenario reduction algorithms in stochastic programming. Comput Optim Appl 24: pp 187206

Hochreiter R, Pflug GC (2007) Financial scenario generation for stochastic multi-stage decision processes as facility location problems. Ann Oper Res 152: pp 257-272

Høyland K, Wallace SW (2001) Generating scenario trees for multistage decision problems. Manage Sci 47(2): pp 295-307

Klaassen P (2002) Comment on generating scenario trees for multistage decision problems. Manage Sci 48: pp 1512-1516

Liu J (2007) Portfolio selection in stochastic environments. Rev Financ Stud 20: pp 1-39

Pflug GC (2001) Optimal scenario tree generation for multiperiod financial planning. Math Program 89(2): pp 25271

Samuelson PA (1969) Lifetime portfolio selection by dynamic stochastic programming. Rev Econ Stat 51: pp 239-246
Wallace SW, Ziemba WT (eds) (2005) Applications of stochastic programming. MPS-SIAM book series on optimization 